\title{
Spectral Analysis and Variable Structural Control of an Elastic Beam
}

\author{
Xuezhang Hou \\ Mathematics Department, Towson University, Baltimore, USA \\ Email: xhou@towson.edu
}

Received September 6, 2013; revised October 8, 2013; accepted October 19, 2013

Copyright (C) 2013 Xuezhang Hou. This is an open access article distributed under the Creative Commons Attribution License, which permits unrestricted use, distribution, and reproduction in any medium, provided the original work is properly cited.

\begin{abstract}
An elastic beam system formulated by partial differential equations with initial and boundary conditions is investigated in this paper. An evolution equation corresponding with the beam system is established in an appropriate Hilbert space. The spectral analysis and semigroup generation of the system operator of the beam system are discussed. Finally, a variable structural control is proposed and a significant result that the solution of the system is exponentially stable under a variable structural control with some appropriate conditions is obtained.
\end{abstract}

Keywords: Spectral Analysis; Semigroups of Linear Operators; Elastic Beam System; Variable Structural Control

\section{Introduction}

A great attention has been paid to the dynamics and control of flexible robot (see [1-5]) in the past thirty years since the high-speed performance and low energy consumption are highly demanded. In this paper, as a continuation of our work [6-9], we shall investigate an elastic robot system formulated by partial differential equations with initial-boundary value conditions. By means of functional analysis and semigroups of linear operators, the beam system is described as an evolution equation in an appropriate Hilbert space. Spectral properties and semigroup generation of the system operator corresponding to the evolution equation are studied. Several significant results are obtained.

Let us consider a robot system composed of two link-arm and three joints, an electrical machinery is installed on each joint, the beam connecting with based stand is rigid and forearm is elastic. By means of the space kinetic and Hamilton's variation principle, we can obtain the following second-order hyperbolic system that describes the motion of the elastic beam system [10]:

$$
\begin{aligned}
& \rho \frac{\partial^{2} u(x, t)}{\partial t^{2}}+\frac{\partial^{2}}{\partial x^{2}}\left(p_{1}(x) \frac{\partial^{2} u(x, t)}{\partial x^{2}}\right) \\
& +\eta \frac{\partial^{2}}{\partial x^{2}}\left(p_{1}(x) \frac{\partial^{3} u(x, t)}{\partial t \partial x^{2}}\right) \\
& =\rho(L \ddot{\varphi}+x \ddot{\theta}+g \cos \theta)
\end{aligned}
$$

$$
\begin{aligned}
& \rho \frac{\partial^{2} v(x, t)}{\partial t^{2}}+\frac{\partial^{2}}{\partial x^{2}}\left(p_{2}(x) \frac{\partial^{2} v(x, t)}{\partial x^{2}}\right) \\
& +\eta \frac{\partial^{2}}{\partial x^{2}}\left(p_{2}(x) \frac{\partial^{3} v(x, t)}{\partial t \partial x^{2}}\right) \\
& =\rho[\ddot{\beta}(L \cos \varphi+x \cos \theta)-x \dot{\beta} \dot{\theta} \sin \theta]
\end{aligned}
$$

with the following boundary conditions:

$$
\begin{gathered}
u(0, t)=u^{\prime}(0, t)=0,\left(p_{1} u^{\prime \prime}\right)(l, t)=0,\left(p_{1} u^{\prime \prime}\right)^{\prime}(l, t)=m g, \\
v(0, t)=v^{\prime}(0, t)=0,\left(p_{2} v^{\prime \prime}\right)(l, t)=0,\left(p_{2} v^{\prime \prime}\right)^{\prime}(l, t)=0,
\end{gathered}
$$

and initial conditions

$$
\begin{aligned}
& u(x, 0)=u_{0}(x), \dot{u}(x, 0)=u_{1}(x) ; \\
& v(x, 0)=v_{0}(x), \dot{v}(x, 0)=v_{1}(x), \\
& \theta(0)=\theta_{0}, \dot{\theta}(0)=\theta_{1} ; \varphi(0)=\varphi_{0}, \\
& \dot{\varphi}(0)=\varphi_{1} ; \beta(0)=\beta_{0}, \dot{\beta}(0)=\beta_{1},
\end{aligned}
$$

where $u(x, t), v(x, t)$ are vertical and horizontal bending vibration displacements of the forearm of the robot respectively; $p_{1}(x)$, and $p_{2}(x)$ are vertical and horizontal local bending rigidity of the forearm of the robot respectively; and $\alpha_{1}, \beta_{1}, \alpha_{2}$, and $\beta_{2}$ are positive constants such that 
$0<\alpha_{i} \leq p_{i}(x) \leq \beta_{i}<+\infty$. $(i=1,2) ; \beta, \phi, \theta$ are the respective base stand azimuth, the angle of elevation-depression of the forearm and the rear-arm of the robot; $\tau_{1}, \tau_{2}, \tau_{3}$ are the control moments of the forces on the electrical machineries installed on the three joints; $l$ and $L$ are the length of the forearm and the rear-arm respectively; $\eta$ is the damping coefficient of the structure; $\rho$ is the line density of the forearm; $m$ is the mass of the tip body; $g$ is the acceleration of gravity; here $\eta, \rho, m, g$ and $I_{1}, I_{2}, I_{3}$ are all positive constants; the symbols $\dot{u}$ and $u^{\prime}$ express $\partial u / \partial t$ and $\partial u / \partial x$ respectively.

\section{Evolution Equation of the Beam System}

We start this section with defining following operators

$$
\begin{gathered}
A_{i} f=\rho^{-1} \frac{\partial^{2}}{\partial x^{2}}\left(p_{i}(x) \frac{\partial^{2} f(x)}{\partial x^{2}}\right), \\
D\left(A_{i}\right)=\left\{f \in H^{4}(0, l) \mid f, f^{\prime}, f^{\prime \prime},\left(p_{i} f^{\prime \prime}\right)^{\prime} \text { are absolutely continuous function on }[0, l],\right. \\
\left.\left(p_{i} f^{\prime \prime}\right)^{\prime \prime} \in L^{2}[0, l], f(0)=f^{\prime}(0)=0,\left(p_{i} f^{\prime \prime}\right)(l, t)=0 .\left(p_{i} f^{\prime \prime}\right)^{\prime}(l, t)=0\right\} \quad(i=1,2)
\end{gathered}
$$

It should be noted that $f$ in $D\left(A_{1}\right)$ satisfying (1) can be written as $f=u-\tilde{u}$, where the function $u$ suits (1) and (3), and the function $\tilde{u}$ suits (3) and following differential equation

$$
\frac{\partial^{2}}{\partial x^{2}}\left(p_{1}(x) \frac{\partial^{2} \tilde{u}}{\partial x^{2}}\right)=0 .
$$

By solving Equation (5) and (3) we find

$$
\tilde{u}(x)=\int_{0}^{x} \int_{l}^{y} m g \frac{z-1}{p_{1}(z)} \mathrm{d} z \mathrm{~d} y .
$$

Obviously, $f$ in $D\left(A_{1}\right)$ must suit Equation (1) if $u$ suits (1) and (3), as well as $\tilde{u}$ suits (5) and (3)

Lemma 2.1 The operators $A_{1}$ and $A_{2}$ are positive self-adjoint operators in $L^{2}[0, l]$, moreover, $A_{1}^{-1}$ and $A_{2}^{-1}$ exist, and they are compact operators.

Proof Apply integration by parts with the definition of $A$ and the boundary conditions included in $D\left(A_{1}\right)$ to find

$$
\begin{aligned}
\left\langle A_{1} f, f\right\rangle & =\int_{0}^{l} \rho^{-1}\left(p_{1}(x) f^{\prime \prime}(x)\right)^{\prime \prime} \overline{f(x)} \mathrm{d} x \\
& =\rho^{-1} \int_{0}^{l}\left(p_{1}(x) f^{\prime \prime}(x)\right)^{\prime} \overline{f^{\prime}(x)} \mathrm{d} x \\
& =\rho^{-1} \int_{0}^{l} p_{1}(x) f^{\prime \prime}(x) \overline{f^{\prime \prime}(x)} \mathrm{d} x \geq 0 .
\end{aligned}
$$

Since $0<\alpha_{1} \leq p_{1}(x) \leq \beta_{1}<\infty$, we have

$$
\left\langle A_{1} f, f\right\rangle \geq \rho^{-1} \alpha_{1}\left\|f^{\prime \prime}\right\|^{2} \geq 0,
$$

and hence, $A_{1}$ is a symmetric operator.

In order to show that $A_{1}$ is self-adjoint, it suffices to show that there is a constant $c>0$ such that $\left\|A_{1} f\right\| \geq c\|f\|, f \in D\left(A_{1}\right)$ (see [11]).

In fact, we can see from (7) that

$$
\left\|A_{1} f\right\|\|f\| \geq\left\langle A_{1} f, f\right\rangle \geq \rho^{-1} \alpha_{1}\left\|f^{\prime \prime}\right\|^{2}
$$

Applying the boundary conditions of $f$ in $D\left(A_{1}\right)$, we can get the inequality [12].

$$
\int_{0}^{l}|f(x)|^{2} \mathrm{~d} x \leq \frac{l^{4}}{12} \int_{0}^{l}\left|f^{\prime \prime}(x)\right|^{2} \mathrm{~d} x,
$$

and hence

$$
\left\|A_{1} f\right\|\|f\| \geq \rho^{-1} \alpha_{1}\left(\frac{12}{l^{4}}\right) \int_{0}^{l}|f(x)|^{2} \mathrm{~d} x=c\|f\|^{2}
$$

where $c=\frac{12 \rho^{-1} \alpha_{1}}{l^{4}}>0$. It follows that

$$
\left\|A_{1} f\right\| \geq c\|f\|,
$$

and so $A_{1}$ is a positively defined self-adjoint operator.

It is easy to see from (8) that $A_{1}^{-1}$ exists. Now set $A_{1} f=g$, and $f=A_{1}^{-1} g$, then (8) gives us

$$
\left\|A_{1}^{-1} g\right\| \leq \frac{1}{c}\|g\|,
$$

this means that mapping $A_{1}^{-1}: H^{4}(0, l) \rightarrow H^{4}(0, l)$ is bounded, and

$$
\left\|A_{1}^{-1}\right\| \leq \frac{1}{c} .
$$

Thus, $A_{1}^{-1}$ is a compact operator by Sobolev embeding theorem [13].

By similar manner, it can be shown that $A_{2}$ is a positively defined self-adjoint operator, and $A_{2}^{-1}$ exists as a compact operator, and the proof is complete.

We now choose Hilbert space $H=L^{2}[0, l] \times L^{2}[0, l]$ as a state space of Equations (1) and (2), on which inner product and norm are defined as follows:

$$
(u, v)_{H}=\left(u_{1}, v_{1}\right)+\left(u_{2}, v_{2}\right), \quad u, v \in H,
$$

here $u=\left(u_{1}, u_{2}\right)^{\mathrm{T}}, \quad v=\left(v_{1}, v_{2}\right)^{\mathrm{T}}, \quad(\cdot, \cdot)$ is the inner product on $L^{2}[0, l]$. Let 


$$
\begin{gathered}
W(t)=\left[\begin{array}{l}
u(t) \\
v(t)
\end{array}\right], F(t)=\left[\begin{array}{c}
L \ddot{\phi}+x \ddot{\theta}+g \cos \theta \\
\ddot{\beta}(L \cos \phi+x \cos \theta)-\dot{\beta}+x \dot{\theta} \sin \theta
\end{array}\right] \\
A=\left[\begin{array}{cc}
A_{1} & 0 \\
0 & A_{2}
\end{array}\right], D(A)=D\left(A_{1}\right) \times D\left(A_{2}\right) .
\end{gathered}
$$

Then the Equations (1) and (2) with the initial-boundary conditions can be written as follows:

$$
\left\{\begin{array}{l}
\ddot{W}(t)+\eta A \dot{W}(t)+A W(t)=F(t) \\
W(0)=W_{0}, \dot{W}(0)=W_{10}
\end{array}\right.
$$

For the sake of establishing an evolution equation of the system (1) and (2), we introduce a Hilbert space $\mathcal{H}=H \times H$, on which inner product is defined as follows:

$$
\langle\vec{u}, \vec{v}\rangle=\left\langle A u_{(1)}, A v_{(1)}\right\rangle_{H}+\left\langle u_{(2)}, v_{(2)}\right\rangle_{H}, \quad \vec{u}, \vec{v} \in \mathcal{H},
$$

where $\vec{u}=\left(u_{(1)}, u_{(2)}\right)^{\mathrm{T}}, \vec{v}=\left(v_{(1)}, v_{(2)}\right)^{\mathrm{T}}$.

$$
\text { Let } \vec{u}=\left(u_{(1)}, u_{(2)}\right)^{\mathrm{T}}, u_{(1)}=W, u_{(2)}=\mathrm{d} W / \mathrm{d} t \text {. }
$$

$$
\mathcal{A}=\left[\begin{array}{cc}
0 & I \\
-A & -\eta A
\end{array}\right], D(\mathcal{A})=D(A) \times D(A), \mathcal{F}=\left[\begin{array}{c}
0 \\
F(t)
\end{array}\right]
$$

then the robot system (9) can be described first-order abstract evolution equation as follows:

$$
\left\{\begin{array}{l}
\frac{\mathrm{d} \vec{u}(t)}{\mathrm{d} t}=\mathcal{A} \vec{u}(t)+\mathcal{F}(t) \\
\vec{u}(0)=\vec{u}_{0}=(W(0), \dot{W}(0))^{\mathrm{T}} .
\end{array}\right.
$$

\section{Spectral Analysis and Semigroup Generation}

We have discussed the spectral properties and semigroup generation of the system operator $\mathcal{A}$ in the system (10), and obtained the following significant results:

Theorem 3.1 The operator $\mathcal{A}$ is an infinitesimal generator of a $C_{0}$-semigroup $T(t)$ on $\mathcal{H}$, and there are constants $M>0$ such that

$$
\|T(t)\| \leq M \mathrm{e}^{-\omega t}
$$

where $-\omega=\sup \{\operatorname{Re} \mu \mid \mu \in \sigma(\mathcal{A})\}<0$

Proof We shall prove Theorem 3.1 in two different cases,

Case 1. $\eta \neq 2 \lambda_{k}^{-\frac{1}{2}}(k=1,2, \cdots)$. For the sake of simplicity, we denote the eigenpairs of $\mathcal{A}$ by $\left\{\sigma_{n}, \vec{e}_{n}\right\}, n=1,2, \cdots$. For every real $\lambda$, $\lambda>-\omega=\sup \{\operatorname{Re} \mu \mid \mu \in \sigma(\mathcal{A})\}$, we see from [11] that $\lambda \in \rho(\mathcal{A})$. For any $\vec{u} \in \mathcal{H}$, since $\left\{\vec{e}_{n}\right\}$ constitutes a
Riesz basis of $\mathcal{H}, \vec{u}=\sum_{n=1}^{\infty} a_{n} \vec{e}_{n}$. A simple computation shows that

$$
(\lambda I-\mathcal{A})^{-1} \vec{u}=\sum_{n=1}^{\infty} a_{n} \frac{1}{\lambda-\sigma_{n}} \vec{e}_{n}
$$

and

$$
\begin{aligned}
& \left\|\left[(\lambda I-\mathcal{A})^{-1}\right]^{m} \vec{u}\right\| \\
& =\left\|\sum_{n=1}^{\infty} a_{n} \frac{1}{\left(\lambda-\sigma_{n}\right)^{m}} \vec{e}_{n}\right\| \\
& =\left\|\sum_{n=1}^{\infty} a_{n} \frac{(\lambda+\omega)^{m}}{\left(\lambda-\sigma_{n}\right)^{m}} \frac{1}{(\lambda+\omega)^{m}} \vec{e}_{n}\right\|
\end{aligned}
$$

It should be noted that $\left|(\lambda+\omega)^{m} /\left(\lambda-\sigma_{n}\right)^{m}\right| \leq 1$ because $-\omega=\sup \{\operatorname{Re} \mu \mid \mu \in \sigma(\mathcal{A})\}$ and $\lambda>-\omega$. Therefore,

$$
\left\|\left[(\lambda I-\mathcal{A})^{-1}\right]^{m} \vec{u}\right\| \leq \frac{1}{(\lambda+\omega)^{m}}\left\|\sum_{n=1}^{\infty} a_{n} \vec{e}_{n}\right\|=\frac{1}{(\lambda+\omega)^{m}}\|\vec{u}\|
$$

We thus arrive at the following result:

$$
\left\|\left[(\lambda I-\mathcal{A})^{-1}\right]^{m}\right\| \leq \frac{1}{(\lambda+\omega)^{m}}, \quad \lambda>-\omega, \quad m=1,2, \cdots
$$

It follows from the theorem 5.3 of [14] that $\mathcal{A}$ is the infinitesimal generator of a $C_{0}$-Semigroup $T(t)$ on $\mathcal{H}$, and $\|T(t)\| \leq M \mathrm{e}^{-\omega t}$, where $M \geq 1$.

Case 2. $\eta=2 \lambda_{k^{*}}^{-\frac{1}{2}}$ for some positive integer $k^{*}$. We see from [11] that for any $\vec{u} \in \mathcal{H}$,

$$
\vec{u}=\sum_{\substack{k=1 \\
k \neq k^{*}}}^{\infty} \sum_{j=1}^{n_{k}}\left(a_{k_{j}}^{(1)} \vec{\phi}_{k_{j}}+a_{k_{j}}^{(2)} \vec{\psi}_{k_{j}}\right)+\sum_{j=1}^{n_{k^{*}}}\left[b_{k_{j}^{*}}^{(1)}\left(\begin{array}{c}
0 \\
\phi_{k_{j}^{*}}
\end{array}\right)+b_{k_{j}^{*}}^{(2)} \vec{\psi}_{k_{j}^{*}}\right]
$$

Since

$$
\mathcal{A} \vec{\psi}_{k_{j}^{*}}=\mu_{k^{*}} \vec{\psi}_{k_{j}^{*}}, \quad j=1,2, \cdots, j_{k^{*}}
$$

and

$$
\begin{aligned}
\mathcal{A}\left(\begin{array}{c}
0 \\
\phi_{k_{j}^{*}}
\end{array}\right) & =\left(\begin{array}{cc}
0 & I \\
-A & -\eta A
\end{array}\right)\left(\begin{array}{c}
0 \\
\phi_{k_{j}^{*}}
\end{array}\right)=\left(\begin{array}{c}
\phi_{k_{j}^{*}} \\
-\eta A \phi_{k_{j}^{*}}
\end{array}\right) \\
& =\left(\begin{array}{c}
\phi_{k_{j}^{*}} \\
-2 \lambda_{k^{*}}^{\frac{1}{2}} A \phi_{k_{j}^{*}}
\end{array}\right)=\left(\begin{array}{c}
\phi_{k_{j}^{*}} \\
-\lambda_{k^{*}}^{\frac{1}{2}} \phi_{k_{j}^{*}}
\end{array}\right)+\left(\begin{array}{c}
0 \\
-\lambda_{k^{*}}^{\frac{1}{2}} \phi_{k_{j}^{*}}
\end{array}\right) .
\end{aligned}
$$


Since $\eta=2 \lambda_{k^{*}}^{-\frac{1}{2}}$, we refer to 1 ) of Theorem 1 to find

$$
\begin{aligned}
\mu_{k^{*}} & =\left(-\eta \lambda_{k^{*}}-\sqrt{\left(\eta \lambda_{k^{*}}\right)^{2}-4 \lambda_{k^{*}}}\right) / 2 \\
& =\left(-2 \lambda_{k^{*}}^{\frac{1}{2}}-\sqrt{\left(2 \lambda_{k^{*}}^{\frac{1}{2}} \lambda_{k^{*}}\right)^{2}-4 \lambda_{k^{*}}}\right) / 2 \\
& =\left(-2 \lambda_{k^{*}}^{\frac{1}{2}}-\sqrt{4 \lambda_{k^{*}}-4 \lambda_{k^{*}}}\right) / 2 \\
& =-2 \lambda_{k^{*}}^{\frac{1}{2}} / 2=-\lambda_{k^{*}}^{\frac{1}{2}},
\end{aligned}
$$

and so

$$
\begin{aligned}
\mathcal{A}\left(\begin{array}{c}
0 \\
\phi_{k_{j}^{*}}
\end{array}\right) & =\left(\begin{array}{c}
\phi_{k_{j}^{*}} \\
\mu_{k_{j}^{*}} \phi_{k_{j}^{*}}
\end{array}\right)+\left(\begin{array}{c}
-\lambda^{\frac{1}{2}} \\
k^{*}
\end{array}\right)\left(\begin{array}{c}
0 \\
\phi_{k_{j}^{*}}
\end{array}\right) \\
& =\sqrt{\lambda_{k^{*}}^{2}+\lambda_{k^{*}}} \vec{\psi}_{k_{j}^{*}}+\left(\begin{array}{c}
-\lambda^{\frac{1}{2}} \\
k^{*}
\end{array}\right)\left(\begin{array}{c}
0 \\
\phi_{k_{j}^{*}}
\end{array}\right) .
\end{aligned}
$$

Hence, the space spanned by

$$
\left\{\vec{\psi}_{k_{1}^{*}}, \cdots, \vec{\psi}_{k_{k_{k}^{*}}^{*}}\right\} \bigcup\left\{\left(\begin{array}{c}
0 \\
\phi_{k_{1}^{*}}
\end{array}\right), \cdots,\left(\begin{array}{c}
0 \\
\phi_{k_{k_{k}^{*}}^{*}}
\end{array}\right)\right\} \text { is an invariant }
$$

subspace of $2 j_{k^{*}}$ dimensions of $\mathcal{A}$, denoted by $\mathfrak{M}_{k^{*}}$. From theory of finite dimensional space, we assert that

$$
\sigma\left(\mathcal{A} \mid \mathfrak{M}_{k^{*}}\right)=\sigma_{p}\left(\mathcal{A} \mid \mathfrak{M}_{k^{*}}\right) \subseteq \sigma_{p}(\mathcal{A}) \subseteq \sigma(\mathcal{A}),
$$

and therefore $-\omega^{*}=\sup \left\{\operatorname{Re} \mu \mid \mu \in \sigma\left(\mathcal{A} \mid \mathfrak{M}_{k^{*}}\right)\right\} \leq$ $\sup \{\operatorname{Re} \mu \mid \mu \in \sigma(\mathcal{A})\}=-\omega$. Actually, we can arrange the vectors spanning $\mathfrak{M}_{k^{*}}$ as follows

$$
\left(\begin{array}{c}
0 \\
\phi_{k_{1}^{*}}
\end{array}\right), \vec{\psi}_{k_{1}^{*}},\left(\begin{array}{c}
0 \\
\phi_{k_{2}^{*}}
\end{array}\right), \vec{\psi}_{k_{2}^{*}}, \cdots,\left(\begin{array}{c}
0 \\
\phi_{k_{j_{k^{*}}^{*}}^{*}}
\end{array}\right), \phi_{k_{j_{k^{*}}^{*}}^{*}} .
$$

Set

$$
\mathbb{A}=\left(\begin{array}{cc}
-\lambda_{k^{*}}^{\frac{1}{2}} & \sqrt{\lambda_{k^{*}}^{2}+\lambda_{k^{*}}} \\
0 & \lambda_{k^{*}}
\end{array}\right),
$$

then $\mathcal{A} \mid \mathfrak{M}_{k^{*}}$ has the form

$$
\begin{aligned}
& \mathcal{A} \mid \mathfrak{M}_{k^{*}} \\
& =\left[\begin{array}{lll}
\mathbb{A} & & 0 \\
& \ddots & \\
0 & & \mathbb{A}
\end{array}\right]\left(\text { there are } j_{k^{*}} \mathbb{A}^{\prime} s\right. \text { in the diagonal) }
\end{aligned}
$$

Apply the result of [14] to conclude that $\mathcal{A}$ generates a $C_{0}$-semigroup $T_{1}(t)$ satisfying $\left\|T_{1}(t)\right\| \leq M_{1} \mathrm{e}^{-\omega^{*} t}$, and so

$$
\left\|T_{1}(t)\right\| \leq M_{1} \mathrm{e}^{-\omega t}
$$

On the other hand, since the family $\left\{\vec{\phi}_{k_{1}}, \vec{\psi}_{k_{1}}, \cdots, \vec{\phi}_{k_{j_{k}}}, \vec{\psi}_{k_{j_{k}}}\right\}_{k \neq k^{*}}$ consists of the eigenvectors of $\mathcal{A}$, the subspace $\mathfrak{M}$ spanned by them is an invariant subspace of $\mathcal{A}$, and this family is just a Riesz basis of $\mathfrak{M}$ [5]. Thus, form case 1 , it is aware of the fast that $\mathcal{A}$ generates a $C_{0}$-Semigroup $T_{2}(t),(t \geq 0)$ in $\mathfrak{M}$. For $\mathfrak{M} \in \rho(\mathcal{A})$, we have

$$
\begin{aligned}
& (\mu I-\mathcal{A})^{-1} \vec{\phi}_{k_{j}}=\frac{1}{\mu-\xi_{k}} \vec{\phi}_{k_{j}}, \\
& (\mu I-\mathcal{A})^{-1} \vec{\varphi}_{k_{j}}=\frac{1}{\mu-\mu_{k}} \vec{\psi}_{k_{j}} \quad\left(j=1,2, \cdots, n_{k} ; k \neq k^{*}\right)
\end{aligned}
$$

and $(\mu I-\mathcal{A})^{-1} \mathfrak{M}_{k} \subset \mathfrak{M}_{k}$, it follows from [16] that $\sigma\left(\mathcal{A} \mid \mathfrak{M}_{k}\right) \subseteq \sigma(\mathcal{A})$ and

$-\omega_{2}=\sup \left\{\operatorname{Re} \mu \mid \mu \in \sigma\left(\mathcal{A} \mid \mathfrak{M}_{k}\right)\right\} \leq$

$\sup \{\operatorname{Re} \mu \mid \mu \in \sigma(\mathcal{A})\}=-\omega$. Thus, there is $M_{2}$ such that

$$
\left\|T_{2}(t)\right\| \leq M_{2} \mathrm{e}^{-\omega t}
$$

Since $\mathfrak{M}_{k^{*}}$ is finite dimensional, it is a closed subspace of $\mathcal{H}^{k}$, and so $\mathcal{H}=\mathfrak{M}_{k^{*}} \oplus \mathfrak{M}_{k}$, where $\oplus$ expresses orthogonal sum in Hilbert space $\mathcal{H}$. Now, we define $T(t) \stackrel{\text { def }}{=} T_{1}(t) \oplus T_{2}(t)$ (obviously,

$\left.T_{1}(t) T_{2}(t)=T_{2}(t) T_{1}(t)=0\right)$. We shall next prove an interesting result that $T(t)$ is exactly a $C_{0}$-semigroup on $\mathcal{H}$ generated by $\mathcal{A}$. The semigroup properties of $T(t)$ can be easily presented as follows:

1) $T(0)=T_{1}(0) \oplus T_{2}(0)=I_{\mathfrak{M}_{k}^{*}} \oplus I_{\mathfrak{M}_{k}}=I_{\mathcal{H}}$

$$
\begin{aligned}
T(t+s) & =T_{1}(t+s) \oplus T_{2}(t+s) \\
& =\left[T_{1}(t) T_{1}(s)\right] \oplus\left[T_{2}(t) T_{2}(s)\right] \\
& =\left[T_{1}(t) \oplus T_{2}(t)\right]\left[T_{1}(s) \oplus T_{2}(s)\right] \\
& =T(t) T(s) \quad(t, s \geq 0)
\end{aligned}
$$

3) For every $\quad x \in \mathcal{H}, \quad x=x_{k^{*}} \oplus x_{k}$, where $\quad x_{k^{*}} \in \mathfrak{M}_{k^{*}}$, $x_{k} \in \mathfrak{M}_{k}$,

$$
\begin{aligned}
\lim _{t \rightarrow 0^{+}} T(t) x= & \lim _{t \rightarrow 0^{+}}\left[T_{1}(t) \oplus T_{2}(t)\right]\left(x_{k^{*}} \oplus x_{k}\right) \\
= & \lim _{t \rightarrow 0^{+}} T(t) x \lim _{t \rightarrow 0^{+}}\left[T_{1}(t) \oplus T_{2}(t)\right] x_{k^{*}} \\
& \oplus\left[T_{1}(t) \oplus T_{2}(t)\right] x_{k} \\
= & \lim _{t \rightarrow 0^{+}}\left[T_{1}(t) x_{k^{*}} \oplus T_{2}(t) x_{k}\right] \\
= & \left(\lim _{t \rightarrow 0^{+}} T_{1}(t) x_{k^{*}}\right) \oplus\left(\lim _{t \rightarrow 0^{+}} T_{2}(t) x_{k}\right) \\
= & x_{k^{*}} \oplus x_{k}=x
\end{aligned}
$$

4) For any $x \in D(\mathcal{A})$, we have $x=x_{k^{*}} \oplus x_{k}$, $x_{k^{*}} \in \mathfrak{M}_{k^{*}}$ and $x_{k} \in \mathfrak{M}_{k}$, and 


$$
\begin{aligned}
\mathcal{A} x= & \mathcal{A}\left(x_{k^{*}} \oplus x_{k}\right)=\mathcal{A} x_{k^{*}} \oplus \mathcal{A} x_{k} \\
= & \left(\lim _{t \rightarrow 0^{+}} \frac{T_{1}(t) x_{k^{*}}-x_{k^{*}}}{t}\right) \\
& \oplus\left(\lim _{t \rightarrow 0^{+}} \frac{\left(T_{1}(t) x_{k^{*}} \oplus T_{2}(t) x_{k^{*}}\right)-\left(x_{k^{*}} \oplus x_{k}\right)}{t}\right) \\
= & \lim _{t \rightarrow 0^{+}} \frac{T x-x}{t}
\end{aligned}
$$

Thus, $T(t)$ defined by the orthogonal sum of $T_{1}(t)$ and $T_{2}(t)$ is exactly $C_{0}$-Semigroup on $\mathcal{H}$ generated by $\mathcal{A}$. Taking $M=\max \left\{M_{1}, M_{2}\right\}$ from (11) and (12), leads to the following result

$$
\|T(t)\| \leq M \mathrm{e}^{-\omega t} \quad(t \geq 0)
$$

The proof of Theorem 11 is complete.

\section{Stabilization with Variable Structural Control}

The variable structural system is a system whose structure is intentionally changed with a discontinuous control and it drives the phase trajectory to a hyperplane or manifold. This method is well-known for its robustness to disturbance and parameter variations [15-18]. Conventionally, the variable structure control is based on the state-space approach in which a Lyapunov function need to be constructed so that the derivative of the Lyapunov function negative definite. As the method provides robustness characteristics, there exists a major problem, that is, the chattering phenomenon, usually encountered in the practical implementation. This phenomenon is highly undesirable because it may excite the high-frequency unmodelled dynamics.

In this section, let us consider the robot system (10) equipped with a feedback controller $w(\vec{u}(t), t)$ :

$$
\left\{\begin{array}{l}
\frac{\mathrm{d} \vec{u}(t)}{\mathrm{d} t}=\mathcal{A} \vec{u}(t)+\mathcal{B} w(\vec{u}, t)+\mathcal{F}(\vec{u}, t) \\
\vec{u}(0)=\vec{u}_{0} .
\end{array}\right.
$$

where $\mathcal{B}$ is a bounded linear operator acting on $\mathcal{H}$ into $\mathcal{H}$. We shall first introduce the equivalent control theorem, and then apply the equivalent control theorem to the robot system to obtain a significant result that the solution of the system is exponentially stable under the variable structural control.

Let now consider the $\delta$-neighborhood of sliding mode $S=C(\vec{u})=0$ where $\delta$ is an arbitrary given positive number, and use a continuous control $\tilde{w}(\vec{u}, t)$ to take place of $w(\vec{u}, t)$ in system (13), we have

$$
\left\{\begin{array}{l}
\frac{\mathrm{d} \vec{u}(t)}{\mathrm{d} t}=\mathcal{A} \vec{u}+\mathcal{B} \tilde{w}(\vec{u}, t)+\mathcal{F}(\vec{u}, t) \\
\vec{u}(0)=\vec{u}_{0}
\end{array}\right.
$$

where $\dot{\vec{u}}=\partial \vec{u} / \partial t$, and the solution of (4.1) belongs to the boundary layer $\|S(\vec{u})\| \leq \delta$.

Let $\dot{S}(\vec{u})=C \dot{\vec{u}}=0$. Applying $C$ to the first equation of (13) leads to the following equivalent control $w_{e q}(\vec{u}, t)$ :

$$
w_{e q}(\vec{u}, t)=-(C \mathcal{B})^{-1} C(\mathcal{A} y+\mathcal{F}(\vec{u}, t)) .
$$

with assumption that the $(C \mathcal{B})^{-1}$ exists. Substituting $u_{e q}(\vec{u}, t)$ into (13) yields

$$
\dot{\vec{u}}=\left[I-\mathcal{B}(C \mathcal{B})^{-1} C\right] \mathcal{A} \vec{u}+\left[I-\mathcal{B}(C \mathcal{B})^{-1} C\right] \mathcal{F}(\vec{u}, t) .
$$

which is called the equivalent control equation.

Denote $P=\mathcal{B}(C \mathcal{B})^{-1} C, \mathcal{A}_{0}=(I-P) \mathcal{A}$ and $\mathcal{F}_{0}(t)=(I-P) \mathcal{F}(\vec{u}, t)$., then (16) is equivalent to the following equation:

$$
\dot{\vec{u}}=\mathcal{A}_{0} \vec{u}+\mathcal{F}_{0}(\vec{u}, t)
$$

We turn now to prove the following result.

Theorem 4.1 If the following conditions are satisfied:

1) $(C \mathcal{B})^{-1}$ exists, and $P \mathcal{A}$ is a closed operator;

2) $\mathcal{F}_{0}(\vec{u}, t)$ satisfies Lipschitz condition in $\vec{u}$ with the constant $L$;

3) the control $w(\vec{u}, t)$ is bounded in any bounded region, and the solution of (13) is unique and bounded in the boundary layer $\|S(\vec{u})\| \leq \delta$; then for each solution $\tilde{\vec{u}}(t)$ of (8) satisfying $S\left(\tilde{\vec{u}}_{0}\right)=0, \tilde{\vec{u}}_{0} \in \dot{D}\left(\mathcal{A}_{0}\right)$, $\left\|\vec{u}_{0}-\tilde{\vec{u}}_{0}\right\| \leq \delta, \quad \vec{u}_{0} \in D(\mathcal{A})$, we have

$$
\lim _{\delta \rightarrow 0}\|\vec{u}(t)-\tilde{\vec{u}}(t)\|=0, \quad \text { uniformly on }[0, T]
$$

Proof Since $P$ is bounded, it is clear that

$$
\|P \mathcal{A} \vec{u}\| \leq\|P\|\|\mathcal{A} \vec{u}\|, \quad \vec{u} \in D(\mathcal{A}) .
$$

Conditions that (ii) together with the above inequality imply that $\mathcal{A}_{0}=\mathcal{A}-P \mathcal{A}$ is an infinitesimal generator of an analytic semigroup $\tilde{T}(t), t \geq 0$ on $\mathcal{H}$ in virtue of [14: Theorem 3.2.1], and so there are constants $N_{1}$, $\omega_{1}, N_{2}, \omega_{2}$, satisfying

$$
\|T(t)\| \leq N_{1} \mathrm{e}^{\omega_{1} t}, \quad\|\tilde{T}(t)\| \leq N_{2} \mathrm{e}^{\omega_{2} t}
$$

In the boundary layer, it is easy to see that

$$
\tilde{w}(\vec{u}, t)=-(C \mathcal{B})^{-1} C(\mathcal{A} \vec{u}+\mathcal{F}(t))+(C \mathcal{B})^{-1} C \dot{\vec{u}}
$$

Substituting (18) into (14), we see that

$$
\begin{aligned}
\dot{\vec{u}} & =(I-P) \mathcal{A} y+(I-P) \mathcal{F}(t)+P \dot{\vec{u}} \\
& =\mathcal{A}_{0} \vec{u}+(I-P) \mathcal{F}(\vec{u}, t)+P \dot{\vec{u}} .
\end{aligned}
$$

and therefore, the solution $\vec{u}(t)$ of (14) can be expressed as follows [14]

$$
\begin{aligned}
\vec{u}(t)= & \tilde{T}(t) \vec{u}_{0}+\int_{0}^{t} \tilde{T}(t-s)(L-P) \mathcal{F}(\vec{u}, s) \mathrm{d} s \\
& +\int_{0}^{t} \tilde{T}(t-s) P \dot{\vec{u}}(s) \mathrm{d} s,
\end{aligned}
$$


the solution $\tilde{\vec{u}}(t)$ of (17) can be written as follows

$$
\tilde{\vec{u}}(t)=\tilde{T}(t) \tilde{\vec{u}}_{0}+\int_{0}^{t} \tilde{T}(t-s)(I-P) \mathcal{F}(\vec{u}, s) \mathrm{d} s .
$$

Employing integration by parts, and estimating the back term of the right side of (19) in view of [14], we obtain

$$
\begin{aligned}
& \int_{0}^{t} \tilde{T}(t-s) P \dot{\vec{u}}(s) \mathrm{d} s \\
= & P \vec{u}(t)-\tilde{T} P \vec{u}_{0}+\int_{0}^{t} \mathcal{A}_{0} \tilde{T}(t-s) P \vec{u}(s) \mathrm{d} s \\
= & P \vec{u}(t)-\tilde{T}(t) P \vec{u}_{0}+\tilde{T}(t) P \vec{u}_{0}-P \vec{u}(t)=0 .
\end{aligned}
$$

Subtract (20) from (19), and employ condition (1) and the inequality $\|\tilde{T}(t)\| \leq N_{2} \mathrm{e}^{\omega_{2} t}$ to find

$$
\|\vec{u}(t)-\tilde{\vec{u}}(t)\| \leq N_{2} \mathrm{e}^{\omega_{2} T} \delta+L N_{2}\|I-P\| \mathrm{e}^{\omega_{2} T} \int_{0}^{t}\|\vec{u}(s)-\tilde{\vec{u}}(s)\| \mathrm{d} s .
$$

The consequence of Theorem 4.1 is now derived from the well-known Gronwall inequality. The proof is complete.

It can be seen from Theorem 4.1 that the robot system (13), and therefore the robot system (10) are exponentially stable under the variable structural control with some appropriate conditions.

\section{Conclusion}

In the present paper, an elastic beam system is described by partial differential equations with initial and boundary conditions investigated. First, an abstract evolution equation is established in an appropriate Hilbert space. Then the spectral analysis and semigroup generation of the system operator of the beam system are studied and applied to prove an equivalent control theorem. Finally, a significant result that the solution of the beam system is exponentially stable under the variable structural control with some appropriate conditions is proved by means of the equivalent control theorem.

\section{REFERENCES}

[1] G. Chen, M. C. Delfour, A. M. Krall and G. Payre, "Modeling Stabilization and Control of Serially Connected Beam,” SIAM Journal on Control and Optimization, Vol. 25, No. 3, 1987, pp. 526-546. http://dx.doi.org/10.1137/0325029

[2] F. Conrad, "Stabilization of Beams by Pointwise Feedback Control," SIAM Journal on Control and Optimization, Vol. 28, No. 2, 1990, pp. 423-437. http://dx.doi.org/10.1137/0328023

[3] Z. H. Luo, "Direct Strain Feedback Control of Flexible Robot Arms: New Theoretical and Experimental Results,” IEEE Transactions on Automatic Control, Vol. 38, No. 11, 1993, pp. 1610-1622. http://dx.doi.org/10.1109/9.262031

[4] B. Z. Guo, "Riesz Basis Approach to Stabilization of a Flexible Beam with a Tip Mass," SIAM Journal on Control and Optimization, Vol. 39, No. 6, 2001, pp. 17361747. http://dx.doi.org/10.1137/S0363012999354880

[5] R. F. Curtain and H. J. Zwart, “An Introduction to Infinite Dimensional Linear System Theory,” Springer-Verlag, New
York, 1995. http://dx.doi.org/10.1007/978-1-4612-4224-6

[6] X. Z. Hou and S.-K. Tsui, "A Control Theory for Cartesian Flexible Robot Arms,” Journal of Mathematical Analysis and Applications, Vol. 225, No. 1, 1998, pp. 265288. http://dx.doi.org/10.1006/jmaa.1998.6027

[7] X. Z. Hou and S.-K. Tsui, "A Mathematical Model for Flexible Robot Arms System Modelling and Optimization,” Chapman \& Hall, Boca Raton, 1999, pp. 391-398.

[8] X. Z. Hou and S.-K. Tsui, "Control and Stability of a Torsional Elastic Robot Arm,” Journal of Mathematical Analysis and Applications, Vol. 243, No. 1, 2000, pp. 140-162. http://dx.doi.org/10.1006/jmaa.1999.6666

[9] X. Z. Hou and S.-K. Tsui, "A Feedback Control and a Simulation of a Torsional Flexible Robot Arm,” Applied Mathematics and Computations, Vol. 142, No. 2-3, 2003, pp. 389-407. http://dx.doi.org/10.1016/S0096-3003(02)00310-7

[10] W. H. Yu, "The Optimal Control for an Elastic Robot System," Control Theorey and Applications, Vol. 12, 1995, pp. 245-258.

[11] X. Z. Hou, "Asymptotical Behavior of a Flexible Robot System with Point-Wise Control,” International Journal of Pure and Applied Mathematics, Vol. 15, No. 3, 2004, pp. 277-295.

[12] A. V. Balaskrishman, “Applied Functinal Analysis,” Springer-Verlag, New York, 1981.

[13] R. A. Adams, "Sobolev Space," Academic Press, New York, 1975.

[14] A. Pazy, "Semigroup of Linear Operators and Applications to Partial Differential Equations," Springer-Verlag, Berlin, 1983.

[15] G. C. Verghese, B. Fernadex and J. K. Hedrick, "Stable Robust Tracking by Sliding Mode Control," Systems \& Control Letters, Vol. 10, No. 1, 1988, pp. 27-34. http://dx.doi.org/10.1016/0167-6911(88)90036-9

[16] K. S. Yeung, C. Cheng and C. Kwan, "A Unifying Design of Sliding Mode and Classical Controllers,” IEEE Transactions on Automatic Control, Vol. 38, No. 9, pp. 1422-1427. http://dx.doi.org/10.1109/9.237660

[17] D. S. Yoo and M. J. Chung, “A Variable Structure Control with Simple Adaptation for Upper Bound on the Norm of the Uncertainties," IEEE Transactions on Automatic Control, Vol. 37, No. 6, 1992, pp. 860-864. http://dx.doi.org/10.1109/9.256348 
[18] S. H. Zak and S. Hui, "On Variable Structure Output Feedback Controllers for Uncertain Dynamic Systems,”
IEEE Transactions on Automatic Control, Vol. 38, 1993, pp. 1509-1512. http://dx.doi.org/10.1109/9.241564 